\title{
Combined Tilt- and Focal-Series Tomography for HAADF-STEM
}

\author{
Tim Dahmen,, ${ }^{*}$ Holger Kohr, ${ }^{2}$ Andrew R. Lupini,, Jean-Pierre Baudoin, ${ }^{5}$ Christian Kübel, ${ }^{6}$ \\ Patrick Trampert, ${ }^{1}$ Philipp Slusallek, ${ }^{1}$ and Niels de Jonge ${ }^{3}$ \\ ${ }^{1}$ German Research Center for Artificial Intelligence (DFKI) GmbH, 66123 Saarbrücken, Germany \\ ${ }^{2}$ KTH Royal Institute of Technology, Lindstedtsvägen 25, Stockholm, SE 100 44, Sweden \\ ${ }^{3}$ INM - Leibniz Institute for New Materials, 66123 Saarbrücken, Germany \\ ${ }^{4}$ Oak Ridge National Laboratory, Materials Science and Technology Division, Oak Ridge, TN 37831-6071 \\ ${ }^{5}$ Aix-Marseille Université, Faculté de Médecine, 27 Bd Jean Moulin, 13005 Marseille, France \\ ${ }^{6}$ Karlsruhe Institute of Technology (KIT), Institute of Nanotechnology (INT) and Karlsruhe Nano Micro Facility (KNMF), 76344 \\ Eggenstein-Leopoldshafen, Germany \\ *tim.dahmen@dfki.de
}

\begin{abstract}
A new aid to tomography in the scanning transmission electron microscope (STEM) is called combined tilt- and focal-series (CTFS). This software controls the recording of a tilt series where for each specimen tilt an entire focal series is recorded. This approach is particularly useful for thick specimens where the tilt range may be limited. Use of CTFS leads to a significant reduction of the missing wedge effect and a better representation of the 3D shapes of features in the specimen.
\end{abstract}

\section{Introduction}

The primary means to study cellular ultrastructure in three dimensions (3D) with nanoscale resolution is currently tilt-series transmission electron microscopy (TEM) [1-2]. In this imaging modality, images are acquired at different projection angles realized by mechanically tilting the sample stage. However, high-quality TEM imaging strongly depends on the condition that the sample is thinner than a few hundreds of nanometers because undesired inelastic and multiple electron scattering can otherwise lead to blurring of the image [3]. For biological materials, sectioning is one way to prepare sufficiently thin samples. It has the disadvantage of limiting the tomogram to a subsection of the sample. For thicker samples containing, for example, whole cells, both inelastic and multiple elastic scattering contribute substantially to the projection data. Scanning transmission electron microscope (STEM), where the sample is scanned by a focused electron beam, is capable of recording images with nanoscale resolution of thicker samples [4-6]. However, because of geometric blurring and scattering of the probe through the sample thickness, resolution decreases with increasing sample thickness. Especially for slablike samples, the effective thickness of the specimen as seen by the electron beam is increasing for higher tilt angles (Figure 1a). For example, a specimen titled to $70^{\circ}$ exhibits a three-fold larger effective thickness than at $0^{\circ}$ tilt. Especially for samples thicker than a few hundred nanometers, this limits the achievable resolution. It thus would be advantageous if the maximum tilt could be limited to $\sim 40^{\circ}$, where the thickness increase is only a factor of 1.3. However, tomographic reconstructions then suffer from severe missing wedge artifacts, visible as elongated objects (axial elongation).

Avoiding tilting altogether is also possible by recording a stack of images with different focal values. Making use of the limited depth of field (DOF) of aberration-corrected STEM, a focal series can be used to obtain 3D STEM information [7-13]. Therein, axial information is retrieved from a stack of images with different focal values. The $3 \mathrm{D}$ data set can be deconvolved to improve the axial resolution and to remove out-of-focus information [14]. However, the focal series data suffers from a vertically elongated point spread function, and the vertical resolution is further reduced by shadowing effects below strongly scattering objects [10-11].

In this article, we present a combined tilt- and focal-series (CTFS) scheme as a new recording method for 3D STEM imaging [15-16]. In this scheme, the specimen is rotated in relatively large tilt increments over a limited tilt range, and for every tilt position a focal series is recorded (Figure 1b). a)

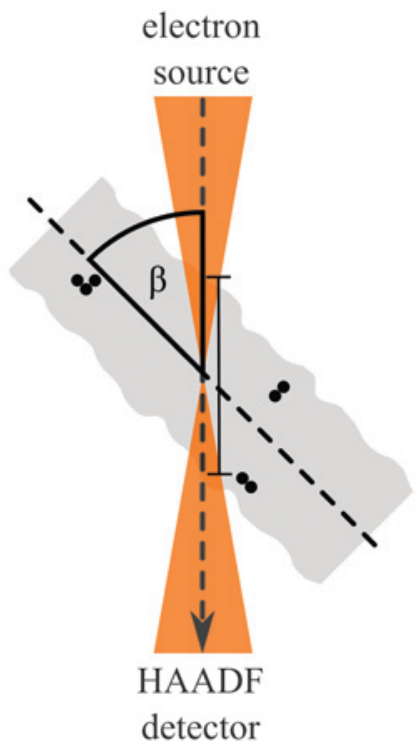

b)

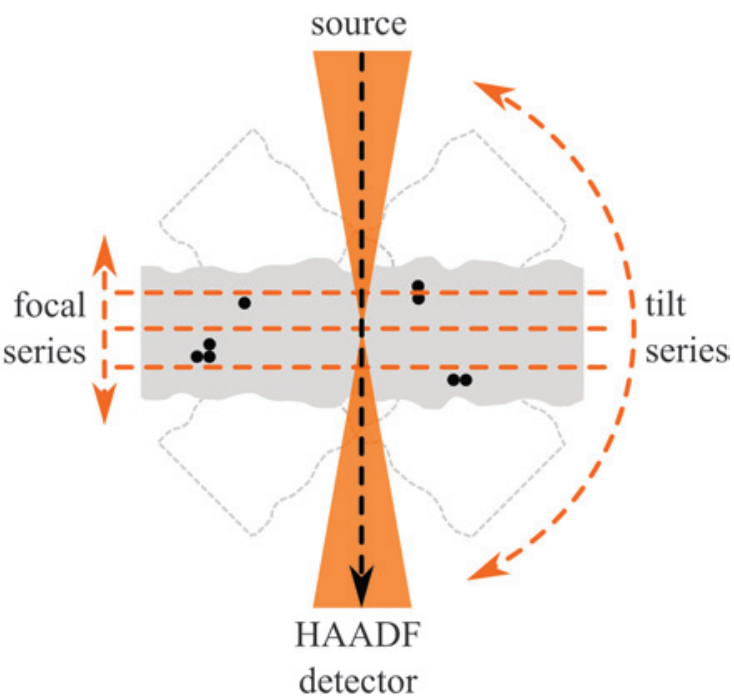

Figure 1: (a) The thickness of the sample as seen by the electron beam increases with $1 / \cos (\beta)$ as the sample is tilted by an angle $\beta$. (b) Schematic overview of the CTFS recording scheme. The sample is tilted by relatively large angular increments, and for every tilt position a focal series is recorded. 


\section{SEE THINGS IN LIQUID YOU NEVER THOUGHT POSSIBLE}

Whether your research involves electrochemistry or only requires liquid heating or flow, Poseidon Select is the specific tool that serves as your single solution for any of these environments. Based on an EDS compatible TEM holder with plug-and-play features, this advantage enables you to begin studying hydrated materials, then as the scope of research evolves, add heating and/or electrochemistry options precisely when desired.

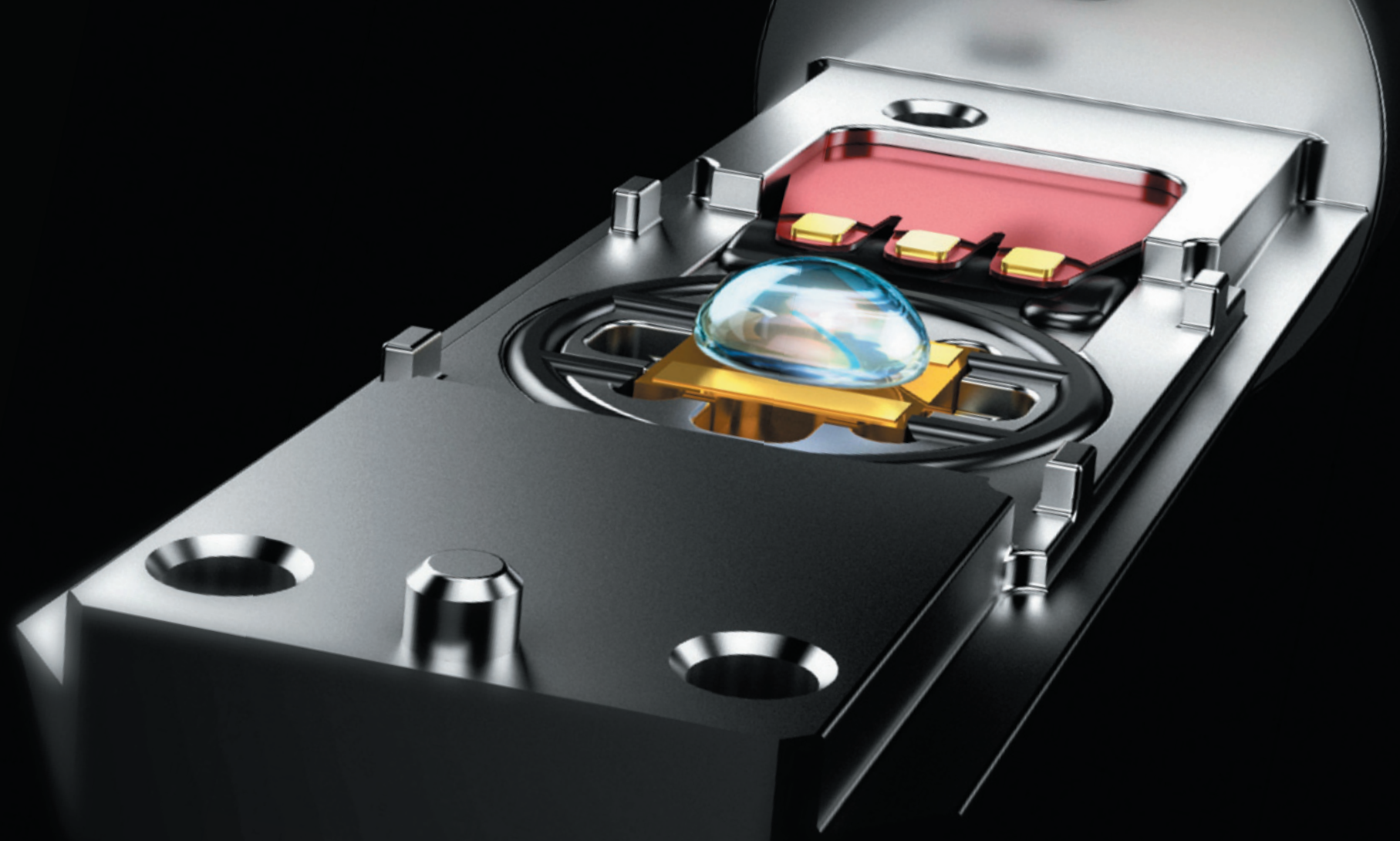


The CTFS method is an alternative to tilt-series STEM tomography, but with improved resolution in the axial direction for a given tilt range.

\section{Materials and Methods}

Image acquisition. To record a CTFS, the objective aperture semiangle $\alpha$ is chosen as high as possible in order to achieve the smallest possible DOF, typically up to about $49 \mathrm{mrad}$ $\left(\approx 2.8^{\circ}\right)$. The available tilt range is then covered in tilt increments of at most $2 \alpha$, that is, the tilt increment is set to $\leq 5.6^{\circ}$. The reason for this condition is that the Fourier transform of a focal series is nonzero in a set that has the shape of an inverted double cone of the opening angle $2 \alpha$ [17]. As long as this condition is fulfilled, the volume
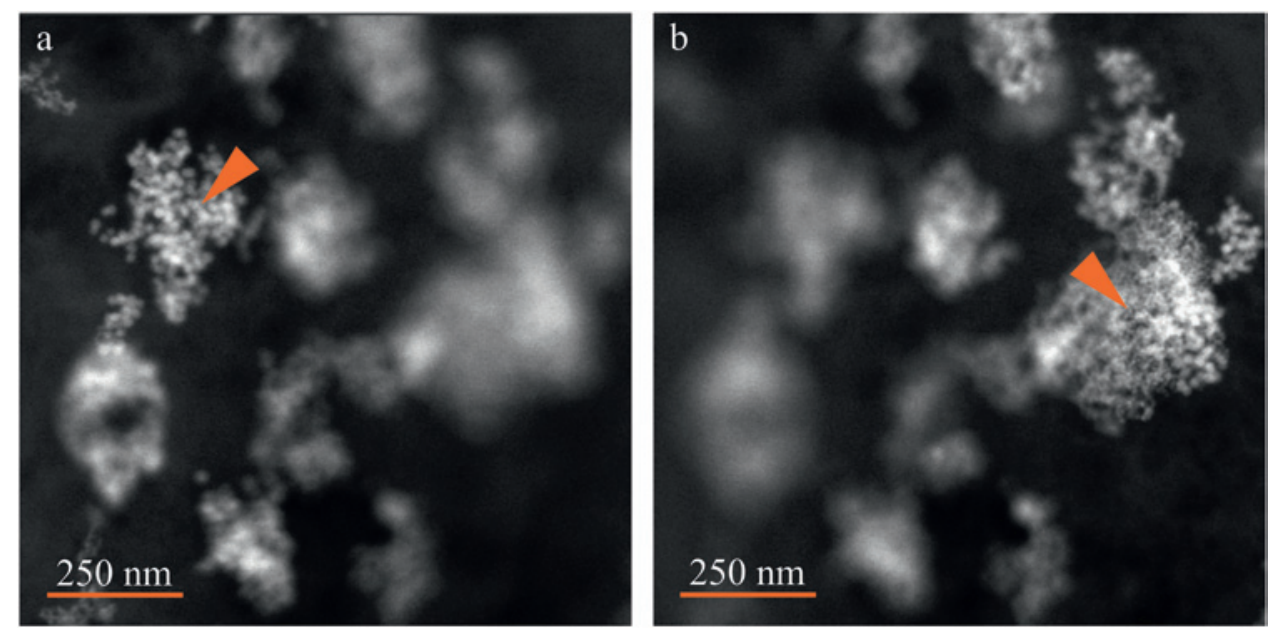

Figure 2: Two images of the CTFS dataset taken at the same tilt angle $\left(-40^{\circ}\right)$ but at different focal values. The difference between the focus planes was $780 \mathrm{~nm}$, which corresponds to 13 images. The orange arrows indicate sections of the images that are in focus. Image adapted from [15]. corresponding to the tilt step is covered in reciprocal space.

For every tilt angle, a focal series is recorded. The distance from one focal plane to the next is selected such that an object perfectly in focus in one focal plane appears blurred by a disc filter of not more than one pixel radius on the next focal plane. This condition ensures that the interpolation error in the backprojection remains small and holds if the distance between consecutive focal planes is $\leq 1 / \sin (\alpha)$.pixelsize. In the case of $\alpha=49 \mathrm{mrad}$, this condition corresponds to a vertical distance of $\approx 20$ pixels.

Data Processing - Alignment. As it is with TEM tilt series, CTFS data need to be aligned to compensate for sample movements during image acquisition. In-plane alignment is initially sufficient between images recorded at the same tilt angle, that is, within a focal stack. If necessary, an additional alignment step can be added to align the images within each focal stack. However, the sample shifts do not only consist of in-plane movements like translation and rotation, but also of an axial shift. The exact focal positions of the images are, thus, unknown.
Lateral alignment errors can be corrected by averaging the images in a focal series and aligning the resulting conventional tilt series using a standard method [18]. In order to compensate for the axial shifts, particles are detected in the tilt series. The particles are tracked through the tilt series, that is, the same particle is identified in several frames of the series [19]. The 3D position of each particle is then computed using triangulation from its position in the different images. In the next step, it is determined in which image of the series the particle is best in focus. The image with the highest intensity at the particle's position is defined as the best focus for this particle and has to be searched in the focal stack. By combining both relations, a given image in a focal series can be assigned a focal depth, and the accuracy of this estimate is improved by averaging over many values gained by repeating this procedure for many particles.

Data Processing - Tomographic Reconstruction. The reconstruction of a $3 \mathrm{D}$ volume from CTFS data is based on an iterative Kaczmarz-type method [20]. It is related to the
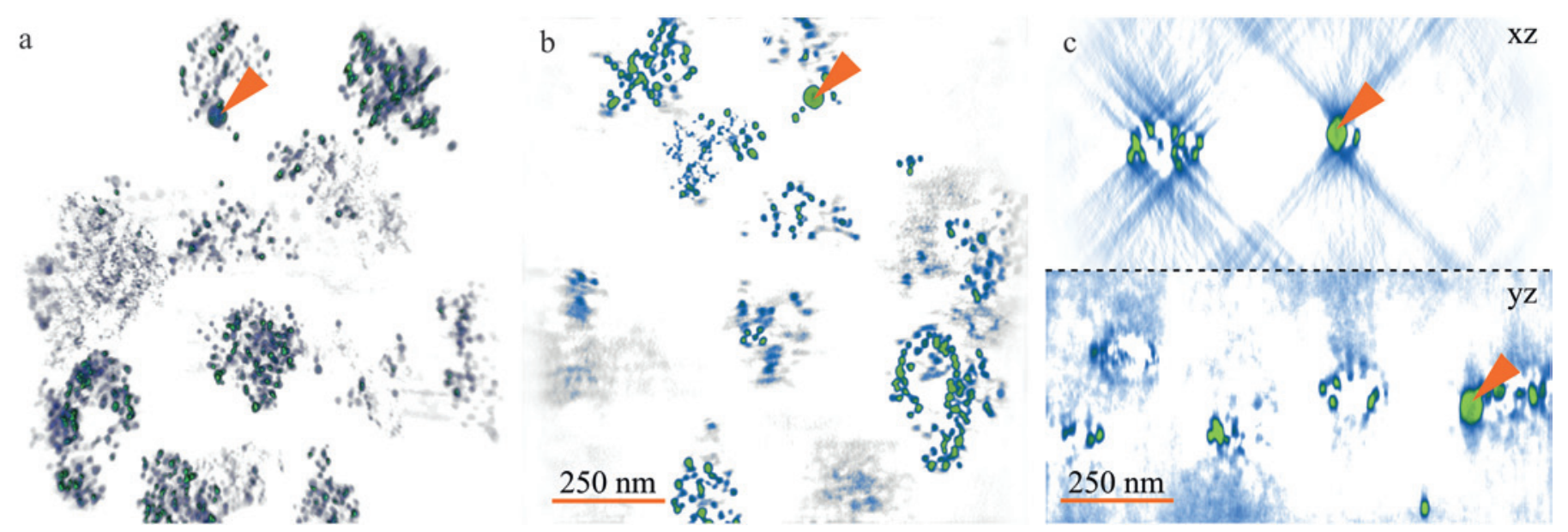

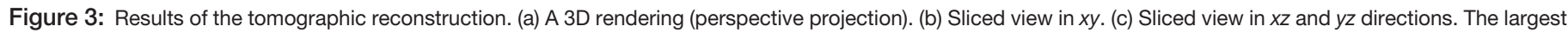
nanoparticle is marked with an orange arrow in all views. All images are coded with false color for better visibility. The slice in $y z$ direction was rotated by $90^{\circ}$. 


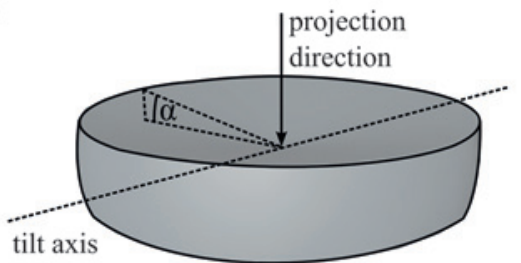

b

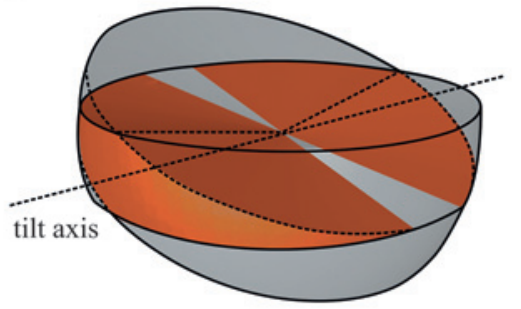

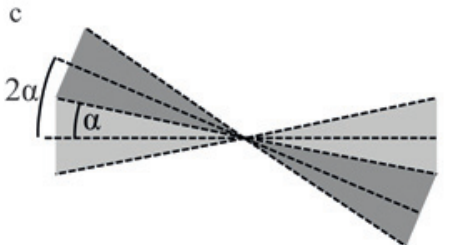

d

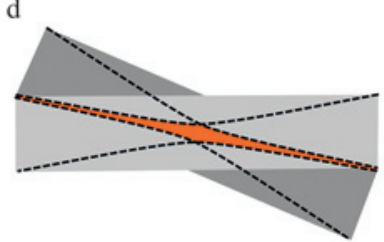

Figure 4: Fourier transform of a focal series. (a) The frequencies covered by one focal stack correspond to the shape of a double-wedge of opening semi-angle $\alpha$. (b) If the tilt increment is chosen as $\Delta \beta=2 \alpha$, neighboring wedges overlap in a non-trivial shape (orange). (c) When considering a cross section through the origin and perpendicular to the tilt axis, the wedges seem to seamlessly cover the entire frequency space. (d) A cross section shifted along the tilt axis reveals a complex-shaped region in frequency space that contains information from more than one tilt direction. (e) A cross section further along the tilt axis toward highest frequencies exposes that the region containing information from both tilt directions expands toward higher frequencies. Image adapted from [17].
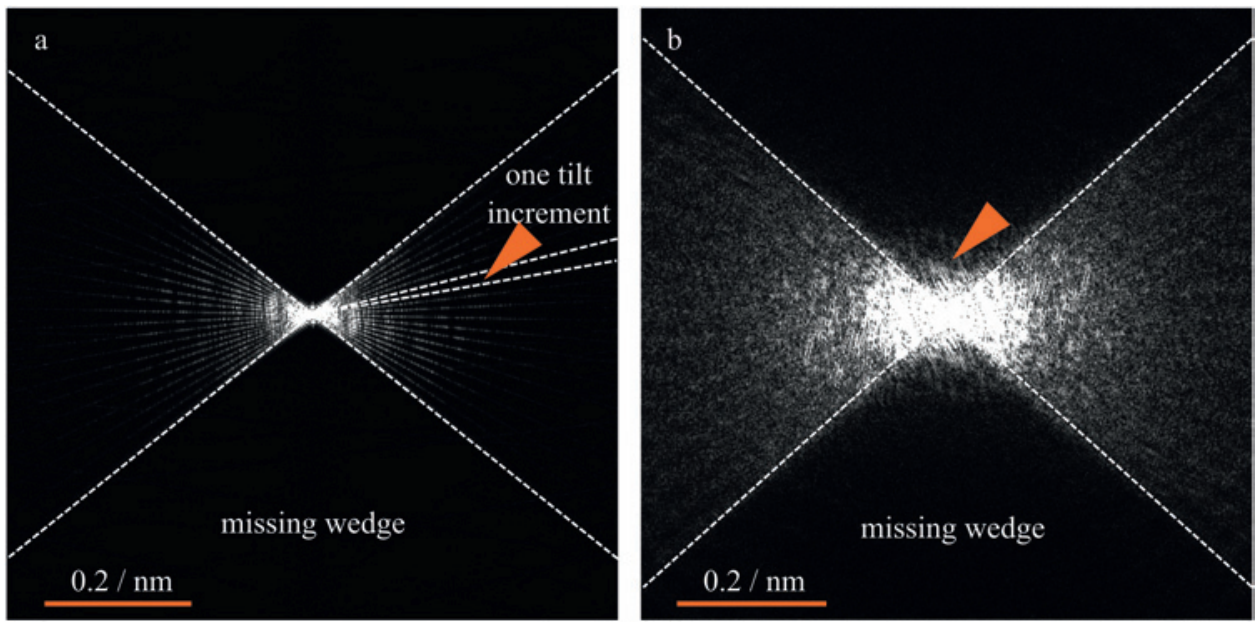

Figure 5: Comparison of tilt-series and CTFS reconstructions using SART. (a) Spatial frequency spectrum (Fourier transform) of an $x z$ slice of the tilt-series reconstruction. The orange arrow indicates a single tilt increment highlighted in the image. (b) Spatial frequency spectrum of an $x z$ slice of the CTFS reconstruction. The orange arrow indicates information reconstructed in part of the missing wedge. The white lines mark the border of the missing wedge. Image adapted from [15].

well-known simultaneous iterative reconstruction scheme (SIRT), but the parallel projection and backprojection models are replaced with weighted integrations of the specimen function over double cones [17]. Thus, the focusing property of a real convergent electron probe is captured in the aberrationcorrected STEM. The forward projection can be computed using a cone-tracing implementation based on stochastic sampling and ray tracing, a technique originally used for the simulation of optical camera systems with limited DOF in movie productions. The backprojection can be implemented based on a convolution with a kernel, which corresponds to a lateral cut through the double cone, that is, a disc. The method is described in detail in [17]. A software implementation is available as a plug-in for the software package Ettention [21]. e

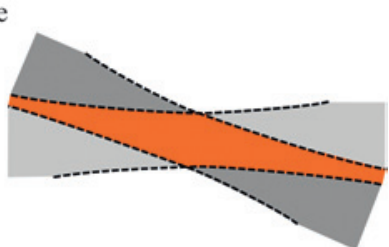

\section{Results}

The method was evaluated on a whole-mount macrophage cell with gold nanoparticles coated with native low-density lipoprotein (LDL) taken up into vesicles [15-22] (Figure 2). The cell had a total thickness of about $1 \mu \mathrm{m}$. The sample was tilted from $-40^{\circ}$ to $+40^{\circ}$ at $5^{\circ}$ increments, resulting in $17 \mathrm{focal}$ stacks. Each stack consisted of 20 images recorded at focal values $60 \mathrm{~nm}$ apart. The entire dataset therefore consisted of 340 images. Each image was recorded at 160,000 magnification with a pixel size of $2.3 \mathrm{~nm}$ and $512^{2}$ pixel resolution. The dataset was aligned as described in the methods section and reconstructed to a tomogram of $512^{3}$ voxels in size (Figure 3). The voxel size of the tomogram was $2.3 \mathrm{~nm}$ in each dimension.

For thick specimens, such as the whole cell investigated in this study, axial elongation as a result of the limited tilt range is the main factor limiting the interpretation of tomograms. In order to quantify axial elongation, we measured the ratio of axial and lateral resolution. Both were determined using the full width at half maximum (FWHM) criterion on a small number of $(n=17)$ spherical particles. The CTFS tomogram had an axial elongation factor $\mathrm{e}_{\mathrm{yz}}=2.2 \pm 0.5$. Measured using the same method, a conventional tilt series recorded at the same tilt range and reconstructed using the SART method has an axial elongation factor of $\mathrm{e}_{\mathrm{yz}}=2.8 \pm 0.5$ [1], so the CTFS leads to a significant $\left(p<7.0 \times 10^{-4}\right)$ reduction of the axial elongation factor.

In order to evaluate the improved axial resolution, it is of interest which frequencies are covered by a focal series, providing a look at the imaging with limited DOF in Fourier space. For the case of a parallel projection with unlimited DOF, the Fourier slice theorem states that the Fourier transform of a single projection contains those frequencies that lie on a plane perpendicular to the projection direction in reciprocal space. Comparably, the Fourier transform of the STEM transform is non-zero in a set with the shape of a complementary double cone of semi-angle $\pi / 2-\alpha$ (Figure 4a) [16-17]. Considering the focal stacks from two adjacent tilt directions, one can see that the double cones overlap in a complex shape (Figure $4 \mathrm{~b}$ ). This means that certain spatial frequencies are contained in more than one projection, which might be the factor that stabilizes the reconstruction process and, thus, leads to improved resolution in the axial direction.

The reduced axial elongation of the CTFS compared to a tilt series is basically a result of additional data acquired in 
the recording scheme. The impact of the CTFS can further be understood by considering the Fourier transform of a reconstructed tomogram. In the case of the tilt series (Figure 5a), sharp streaks are present corresponding to the tilt directions. The missing wedge effect is also clearly visible, and the sequential algebraic reconstruction technique (SART) algorithm used to compute the tomogram can reconstruct almost no information in the missing wedge region. In the case of the CTFS method (Figure 5b), the streaks corresponding to the tilt directions are less pronounced and spread over an angular region equal to the beam-opening angle, so additional information is present between the tilt directions. The missing wedge is still present in the CTFS recording, but in the central vertical region low spatial frequency signal components are now present (orange arrow).

\section{Discussion}

Alternative approaches exist to reduce axial elongation for situations with a very limited tilt range using prior knowledge reconstructions. Making the assumption that the gradient of the reconstruction volume is sparse, one can formulate reconstruction algorithms that generate only a finite number of different gray values, for example discrete algebraic reconstruction technique (DART) [23] or compressed sensing approaches [24]. These approaches are independent of the CTFS recording scheme. We expect that new algorithms using a sparse gradient assumption for the reconstruction of CTFS data will be developed in the future.

\section{Conclusion}

For the imaging of thick samples of $0.5 \mu \mathrm{m}$ or more, the HAADF-STEM mode is a powerful alternative to conventional TEM. Because aberration-corrected STEM images have a very limited DOF, tilt-series STEM tomography can be supplemented by depth sectioning. This approach helps to gather $3 \mathrm{D}$ information, particularly in situations where the accessible tilt range is very limited, as it is typically the case for very thick specimens. The recording scheme is called CTFS and consists of a tilt series, where for each tilt direction an entire focal series is recorded. Reconstructions from CTFS data were reconstructed using an iterative reconstruction algorithm that considered the convergent nature of the electron beam in the forward model. The CTFS led to a significant reduction of the axial elongation, a significant reduction of the missing wedge effect, and a better representation of the $3 \mathrm{D}$ shape of objects in the sample.

\section{Acknowledgements}

This research was partially funded by the European Research Project NOTOX (FP7-267038) and the DFG grant IMCL (AOB): 600875). The authors thank Eduard Arzt for his support through INM and the DFKI GmbH for additional funding. The authors acknowledge the electron microscopy supported by the Materials Sciences and Engineering Division, Basic Energy Sciences, Office of Science, U.S. Department of Energy (ARL), using instrumentation at the Oak Ridge National Laboratory's Center for Nanophase Materials Sciences (CNMS), which is sponsored by the Scientific User Facilities Division, Office of Basic Energy Sciences, U.S. Department of Energy, as well as the electron microscopy support provided by the Karlsruhe Nano Micro Facility (KNMF), a Helmholtz large-scale user facility operated at the Karlsruhe Institute of Technology (KIT).

\section{References}

[1] A Hoenger and JR McIntosh, Curr Opin Cell Biol 21 (2009) 89-96.

[2] LF Kourkoutis et al., Ann Rev Mater Res 42 (2012) 33-58.
[3] V Lucić et al., Annu Rev Biochem 74 (2005) 833-65.

[4] C Kübel et al., Microsc Microanal 11 (2005) 378-400.

[5] MF Hohmann-Marriott et al., Nat Meth 6 (2009) 729-31.

[6] K Aoyama et al., Ultramicroscopy 109 (2008) 70-80.

[7] K Van Benthem et al., Appl Phys Lett 87 (2005) 1-3.

[8] SP Frigo et al., Appl Phys Lett 81 (2002) 2112.

[9] AY Borisevich et al., Proc Natl Acad Sci USA 103 (2006) 3044-48.

[10] G Behan et al., Phil Trans A, Math Phys Eng Sci 367 (2009) 3825-44.

[11] N de Jonge et al., Microsc Microanal 16 (2010) 54-63.

[12] MJ Dukes et al., J Struct Biol 174 (2011) 552-62.

[13] W Van den Broek et al., Ultramicroscopy 141 (2014) 22-31.

[14] R Ramachandra and N de Jonge, Microsc Microanal 18 (2012) 218-28.

[15] T Dahmen et al., Microsc Microanal S3 20 (2014) 1-13.

[16] R Hovden et al., Ultramicroscopy 140 (2014) 26-31.

[17] T Dahmen et al., Microsc Microanal 21 (2015) 725-38.

[18] JR Kremer et al., J Struct Biol 116 (1996) 71-76.

[19] COS Sorzano et al., BMC Bioinformatics 10 (2009) 124-35.

[20] F Natterer, The Mathematics of Computerized Tomography, Society for Industrial and Applied Mathematics, Philadelphia, 2001.

[21] T Dahmen et al., at Microscopy \& Microanalysis 2015, Portland, OR.

[22] J-P Baudoin et al., PLoS One 8 (2013) e55022-1-8.

[23] K Batenburg and J Sijbers, Image Processing 2007, ICIP 2007, 4(2) (2007) IV 133.

[24] R Leary et al., Ultramicroscopy 131 (2013) 70-91. MT

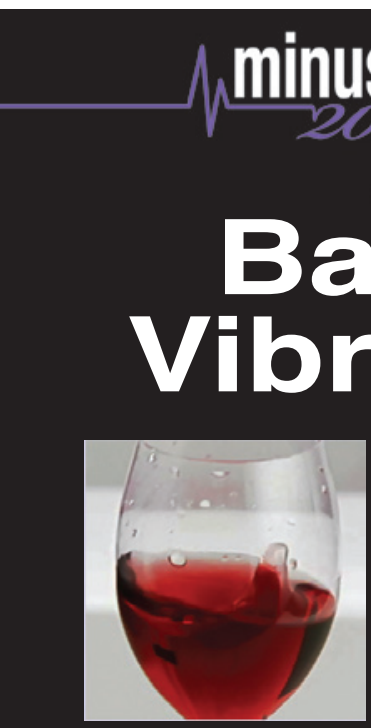

Without Minus $\mathbf{K}^{\circledR}$ 
The moment "I think" becomes "I know".

\section{This is the moment we work for.}

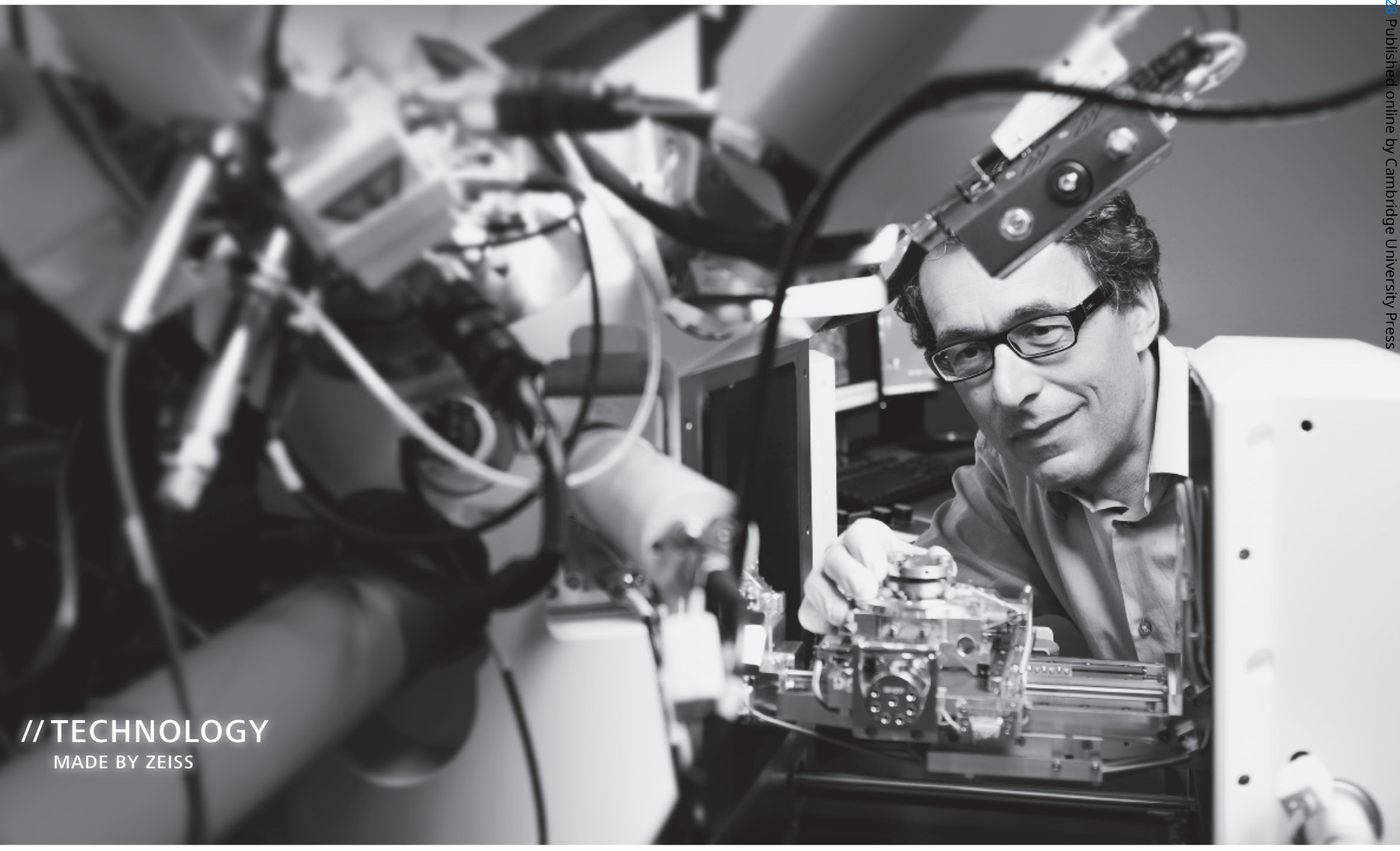

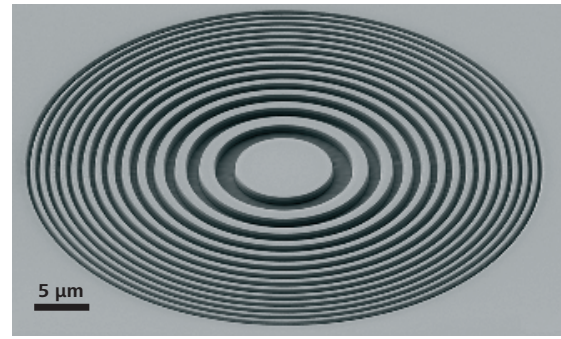

A Fresnel zone plate is optical device which can be used as a lens for extremely short wavelengths. Large area imaging (32K $\times 32 \mathrm{~K})$ with ZEISS ATLAS 5.

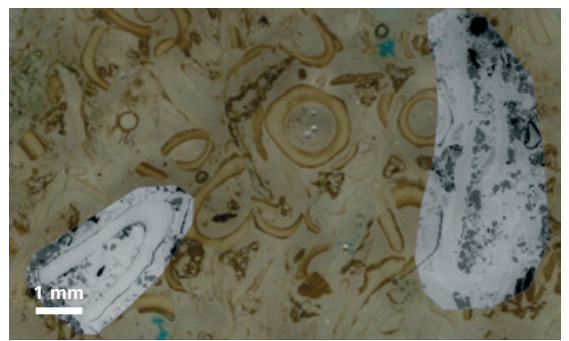

Using ZEISS Atlas 5 software, a light microscope overview image (brown) is correlated with high resolution SEM images (gray) for a fossiliferous carbonate sandstone sample. Sample courtesy of $D$. Schumann, Fibics Incorporated, Ottawa, Canada.

\section{Master Your Multiscale Challenge with ZEISS Atlas 5}

Acquire large sets of 2D or 3D nanoscale electron microscope (EM) images for hours, or even days, without operator supervision. Atlas 5 's correlative workspace makes it easy to bring together images from multiple sources: zoom in from the full macroscopic view of your sample down to nanoscale details. Understand your sample fully in both 2D and 3D - Atlas 5 employs a novel graphical user interface concept that makes it easy 